\title{
Otimismo, Autoeficácia e Lócus de Controle na Adesão ao Tratamento de Pessoas Hipertensas
}

\author{
Optimism, Self-Efficacy and Locus of Control on Hypertension Treatment Adherence
}

Optimismo, Autoeficacia y Locus de Control en la Adherencia al Tratamiento em Hipertensos

\author{
Lucila Moraes Chaves Santos \\ Universidade Federal de Sergipe ${ }^{1}$ \\ Laís Gabriela Rocha Almeida \\ Universidade da Beira Interior-Portugal \\ André Faro \\ Universidade Federal de Sergipe
}

\begin{abstract}
Resumo
Este estudo analisou a relação do otimismo, da autoeficácia e do lócus de controle na adesão ao tratamento de pessoas hipertensas, bem como os principais preditores da adesão na amostra selecionada, considerando-se as variáveis sociodemográficas e os construtos psicológicos avaliados. A amostra foi composta de 100 hipertensos, e foram utilizados cinco instrumentos: questionário sociodemográfico e clínico, a Escala de Adesão Terapêutica de Morisky, o Teste de Orientação na Vida, a Escala de Autoeficácia Geral Percebida e a Escala de Lócus de Controle da Saúde. Por meio da técnica de regressão logística, verificou-se que praticar atividade física, não consumir álcool, ter maiores pontuação no lócus de controle acaso e na autoeficácia foram preditores de média e alta adesão ao tratamento. Finalmente, os resultados demonstraram a necessidade de mais investigações acerca da relação entre variáveis psicológicas e aderência ao tratamento hipertensivo, especialmente no que se refere ao lócus de controle acaso e sua influência sobre os comportamentos de saúde.
\end{abstract}

Palavras-chave: adesão ao tratamento, otimismo, autoeficácia, lócus de controle, hipertensão

Abstract
This study analyzed the association between optimism, self-efficacy and locus of control on treatment adherence of hypertensive adults, as well as the main predictors of adherence from sociodemographic variables and the psychological constructs. The sample consisted of 100 hypertensive patients. Five instruments were applied: sociodemographic and clinical questionnaire, Morisky Medication Adherence Scale, Life Orientation Test, General Self-Efficacy Scale, and Multidimensional Health Locus of Control Scale. The logistic regression technique showed that physical activity, not consuming alcohol, and having greater scores in luck locus of control and in the self-efficacy were predictors of medium and high adherence. Finally, results demonstrated the need for further investigations on the relationship between psychological variables and adherence to hypertensive treatment, especially about the influence of the locus of control related to the luck on the health behavior.

Keywords: treatment adherence, optimism, self-efficacy, locus of control, hypertension

\section{Resumen}

Esta investigación examinó la relación entre optimismo, autoeficacia y locus de control en la adherencia de las personas hipertensas, así como sus principales predictores, teniendo en cuenta aspectos sociodemográficos y psicológicos evaluados. La muestra fue compuesta de 100 hipertensos. Se utilizaron cinco instrumentos: cuestionario socio demográfico y clínico, Escala de Adherencia al Tratamiento de Morisky, Test de Optimismo, Escala de Autoeficacia Generalizada y Escala Multidimensional de Locus de Control de la Salud. A través de la técnica de regresión logística se verifico que practicar actividad física, no consumir alcohol y tener mayores puntuaciones en locus de control acaso y la autoeficacia fueron predictores de media y alta adhesión. Finalmente, los resultados demostraron la necesidad de mayores investigaciones acerca de la relación entre variables psicológicas y adherencia al tratamiento, especialmente en lo que se refiere al locus acaso y su influencia sobre los comportamientos de salud.

Palabras clave: adherencia al tratamiento, optimismo, autoeficacia, locus de control, hipertensión

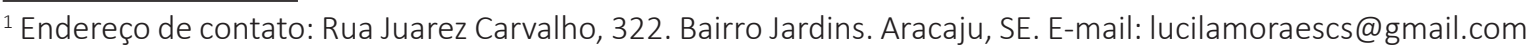




\section{Introdução}

De acordo com a Organização Mundial da Saúde (OMS, 2013), a Hipertensão Arterial Sistêmica (HAS) é caracterizada como uma doença cardiovascular crônica, na qual os vasos sanguíneos estão constantemente sob alta pressão, o que provoca seu estreitamento e faz com que coração necessite de maior força para bombardear o sangue pelo corpo (OMS, 2014). A etiologia da doença é multicausal, ou seja, resulta da interação de fatores genéticos e ambientais tais como o consumo excessivo de sal, o sobrepeso, o sedentarismo e o desgaste psicológico contínuo (Sociedade Brasileira de Cardiologia, 2016).

A HAS é responsável direta ou indiretamente por cerca de 7,1 milhões de mortes no mundo anualmente e atinge um em cada quatro adultos, o que equivale a um bilhão de indivíduos (Joint National Committee. . ., 2004). A OMS (2014) aponta a HAS como causa de $58,7 \%$ das mortes, e espera-se que esse número cresça em decorrência do envelhecimento da população e da maior prevalência dos fatores de risco (Brito \& Giorgi, 2012). Apesar da gravidade dos seus efeitos, a HAS é facilmente diagnosticada, seu tratamento consiste em mudanças de hábitos e, quando necessário, tratamento medicamentoso. As principais modificações aludem aos hábitos, principalmente à redução da ingestão de sal e gorduras, à prática de atividades físicas e ao controle da ingestão do álcool. Entretanto mais de $40 \%$ dos hipertensos não realizam nenhum tratamento e apenas 30\% estão com os níveis pressóricos controlados (Nakamoto, 2012; Ortega, Silva, \& Mion, 2006). Nesse contexto, o desenvolvimento de estratégias para aumentar a adesão ao tratamento dos pacientes é o principal desafio para a efetivação dessas mudanças comportamentais. A adesão ao tratamento foi definida por Afonso (2004) como um comum acordo entre o profissional de saúde e o paciente, em que este último se compromete a realizar as mudanças comportamentais necessárias para a sua melhora e detém participação ativa no tratamento.

Estudos apontam que cerca da metade dos hipertensos diagnosticados abandonam o tratamento no primeiro ano, número que pode chegar a $75 \%$ de abandono após cinco anos (Péres, Magna, \& Viana, 2003). A não adesão ao tratamento pode trazer diversas consequências danosas, dentre elas: surgimento de outras doenças cardíacas mais complexas, o aumento do gasto em remédios e a necessidade de afastamento do trabalho (Araújo \& Garcia, 2006). Dessa forma, a adesão ao tratamento constitui uma importante ferramenta para meIhor entender e intervir no controle pressórico e na qualidade de vida dos hipertensos.

A alta taxa de abandono do tratamento dos pacientes hipertensos pode estar relacionada a variados fatores, como, por exemplo, fatores psicológicos relacionados ao modelo de crenças em saúde (Lima, Meiners, \& Soler, 2010). Esse modelo aborda quais fatores cognitivos e comportamentais influenciam a adesão, levando em conta o efeito das consequências e expectativas relacionadas a ela (Ortiz \& Ortiz, 2007). Sendo assim, fatores psicológicos, como otimismo, autoeficácia e lócus de controle, são capazes de explicar o porquê de alguns indivíduos seguirem as recomendações terapêuticas e outros não.

O otimismo é uma variável psicológica que geralmente está relacionada à tomada de medidas de proteção à saúde física e mental. Portanto pessoas otimistas tendem a agir de forma confiante e persistente frente a um desafio (Bastianello \& Hutz, 2015). As principais contribuições do otimismo na adesão ao tratamento remetem ao fato de que as emoções positivas elevam os recursos sociais, físicos e cognitivos, auxiliando os indivíduos a lidarem 
melhor com situações adversas e preservando a saúde (Straub, 2014). Isso ocorre devido aos otimistas tenderem a adotar estratégias positivas para resolução de problemas e conseguirem se reestruturar cognitivamente com mais facilidade quando comparados com pessoas pessimistas (Carver \& Connor-Smith, 2010). Essa variável tem sido relacionada a efeitos favoráveis no processo de doença, aumentando o tempo de vida em pacientes terminais e incidindo sobre a percepção de bem-estar e saúde dos pacientes (Bernard, 2011). Um estudo realizado com pacientes cardíacos em um programa de reabilitação, por exemplo, mostrou que os pacientes otimistas tinham maior probabilidade de reduzir níveis de gordura saturada e gordura corporal, além de praticarem mais exercício físico (Shepperd, Maroto, \& Pbert, 1996). Com isso, espera-se que, a partir da análise do otimismo dos hipertensos, possa ser estabelecida uma relação entre expectativas positivas da terapêutica e uma melhor adesão ao tratamento, o que permitiria entender e adotar melhores estratégias para enfrentamento da doença.

A autoeficácia é outro construto relacionado a resultados positivos em saúde e diz respeito às crenças dos indivíduos na sua capacidade de planejar e executar um plano de ações para atingir uma meta específica, ou seja, na capacidade de exercer controle sobre o ambiente e as experiências vividas (Bandura, 1977; Castro, Ponciano, \& Pinto, 2010). Altos níveis de autoeficácia facilitam o desempenho e o enfrentamento diante de eventos estressores, diminuindo a vulnerabilidade do indivíduo ao estresse e à depressão. Quanto mais forte for a autoeficácia percebida, mais ousadas tendem a ser as metas estabelecidas e mais seguramente as pessoas tendem a se comprometer com elas (Wood \& Bandura, 1989). Os indivíduos com mais alta autoeficácia percebida têm mais probabilidade de iniciar cuidados preventivos, buscar tratamentos com antecedência e serem mais otimistas em relação a eficácia destes. No estudo de Morowatisharifabad, Ghofranipour, Heidarnia e Ruchi (2006), foi encontrada forte correlação entre autoeficácia e promoção de comportamentos de saúde, principalmente em relação à atividade física, alimentação e gestão do estresse. Assim, a autoeficácia pode ser um importante e modificável preditor para adoção de comportamentos pró-saúde, pois, quando entende que possui recursos suficientes para lidar com sua doença, o indivíduo tem mais chances de procurar maneiras eficazes para seu tratamento (Baptista, Santos, \& Dias, 2006).

Outro construto associado à adesão é o lócus de controle, que diz respeito a como os indivíduos percebem a origem dos eventos que ocorrem em suas vidas como causas internas - causadas pelo próprio sujeito - ou externas - causadas por algum elemento exterior a eles, como Deus, sorte ou o acaso. Rodrigues e Pereira (2007) definiram o lócus de controle como multidimensional, propondo três tipos de orientações para o fenômeno: (a) Internos autênticos, pessoas que não acreditam no controle que outras pessoas ou entidades possuem sobre as situações que ocorrem na vida; (b) Externos defensivos, indivíduos que percebem outras pessoas como controladoras dos eventos que ocorrem consigo, porém não acreditam no poder do acaso/sorte; e, por fim, (c) Externos autênticos, sujeitos que apenas acreditam no poder do acaso ou sorte como controlador das situações que ocorrem em suas vidas.

Estudos apontam que indivíduos com um alto lócus de controle interno tendem a adotar condutas mais benéficas para a saúde - como o desenvolvimento de habilidades de autogerenciamento - ao passo que pessoas com maior lócus de controle externo em saúde pos- 
suem mais dificuldade em seguir orientações médicas e necessitam de um maior tempo de internação (Bernard, 2011; Nazareth et al., 2015). Logo, entender a orientação da percepção de controle do paciente hipertenso auxilia o terapeuta a compreender o quão comprometido o cliente está com a terapia e, com isso, modificar sua percepção para que se alcancem as metas desejadas (Omeje \& Nebo, 2011). Em geral, pesquisas sugerem que indivíduos otimistas, autoeficazes e com uma alta percepção de controle interno geralmente são mais saudáveis, menos suscetíveis a ansiedade, estresse e morte por doenças cardiovasculares. Somado a isso, possuem um melhor controle pressórico e, consequentemente, maior empenho em melhorar suas condições de saúde (Castro et al., 2010; Straub, 2014). Portanto, diante dessas evidências empíricas, o presente estudo torna-se relevante, pois, ao serem identificadas as variáveis que influenciam a adesão ao tratamento hipertensivo, será possível desenvolver estratégias de intervenções comportamentais e cognitivas eficazes junto a tal grupo, tendo em vista que o sucesso no tratamento hipertensivo reside na capacidade de adaptação e aceitação do paciente ao novo estilo de vida imposto pela doença.

Diante disso, o objetivo principal desta pesquisa foi analisar a relação entre o otimismo, a autoeficácia e o lócus de controle na adesão ao tratamento de pessoas hipertensas. Ademais, buscou-se identificar os principais preditores da adesão na amostra pesquisada, considerando-se variáveis sociodemográficas, clínicas e relativas aos construtos psicológicos então estudados.

\section{Método}

\section{Participantes}

A amostra foi composta por 100 hipertensos de ambos os sexos, na faixa etária de 32 a 78 anos. A seleção dos participantes consistiu em amostragem por conveniência, de caráter acidental. A abordagem foi feita no município de Campo do Brito, SE, quando os pacientes estavam à espera da consulta de rotina nas Unidades Básicas de Saúde do município e dos povoados vizinhos. Além desses, foram convidados participantes do projeto Academia da Cidade, bem como moradores de casas próximas à realização dessa atividade. Os critérios de inclusão foram: ser adulto, hipertenso e fazer tratamento medicamentoso.

\section{Instrumentos}

O questionário sociodemográfico abordou informações como a idade, sexo, relacionamento, ofício, escolaridade, nível de religiosidade e renda familiar média nos últimos três meses. Foram realizadas perguntas sobre a HAS, tais como tempo de diagnóstico da doença, tempo do uso de medicação para a HAS, medicações utilizadas, presença de outras comorbidades, valor da pressão arterial na última aferição, status autodeclarado de saúde e doença (atribuição de nota que varia de 0 - doente, a 10 - saudável). Foram ainda realizadas perguntas sobre hábitos de saúde, a saber: peso e altura para cálculo do Índice de Massa Corporal (IMC), prática de atividades físicas, quais atividades físicas, tabagismo e consumo de bebida alcoólica.

Para mensurar a adesão ao tratamento, foi utilizada a Escala de Adesão Terapêutica de Morisky de oito itens (MMAS-8), que foi traduzida do inglês e validada para o Brasil por 
Oliveira-Filho, Barreto-Filho, Neves e Lyra (2012). Essa versão é composta por sete perguntas relacionadas a comportamentos de adesão que podem ser respondidas com sim ou não, e uma pergunta que pode ser respondida com cinco opções (nunca, quase nunca, às vezes, frequentemente e sempre). Os itens abordam o comportamento de tomar a medicação hipertensiva como, por exemplo, "Você às vezes esquece de tomar os seus remédios para pressão?" e "Quando sente que sua pressão está controlada, você às vezes para de tomar seus medicamentos?". Com relação à consistência interna da escala, foi encontrado um alfa de Cronbach de 0,69, considerado como limítrofe, mas aceitável (Hair, Black, Babin, Anderson, \& Tatham, 2009). Originalmente, o grau de adesão do paciente pode ser classificado de acordo com a pontuação adquirida na escala: alta adesão (8 pontos), média adesão ( 6 a $<8$ pontos) e baixa adesão ( $<6$ pontos).

Para medir o otimismo, foi utilizado o Teste de Orientação na Vida (TOV-R), traduzido e validado por Bandeira, Bekou, Lott, Teixeira, \& Rocha (2002), que é composto por dez itens, dos quais quatro são distratores, e seis avaliam o otimismo disposicional. O alfa de Cronbach encontrado para essa escala foi de 0,68, considerado como aceitável (Hair et al., 2009). Seus itens medem a percepção da vida, incluindo afirmações como "É fácil para mim relaxar" e "Raramente eu espero que coisas boas aconteçam comigo". O teste apresenta respostas numa escala de cinco pontos entre "concordo totalmente" e "discordo totalmente", para obter o escore os pontos são somados de forma a indicar que uma pontuação alta demonstra maior otimismo (Bandeira et al., 2002; Gaspar, Ribeiro, Matos, Leal, \& Ferreira, 2009).

Para avaliar a autoeficácia, foi utilizada a Escala de Autoeficácia Geral Percebida, adaptada e validada por Souza e Souza (2004). No que se refere à consistência interna do instrumento, foi encontrado um valor alfa de Cronbach de 0,81, considerado alto (Hair et al., 2009). Ela é composta por dez itens com respostas do tipo Likert de cinco pontos, em que cada item aborda o alcance de metas e insinua uma boa atribuição interna de sucesso, através de itens como "Tenho confiança para me sair bem em situações inesperadas" e "Se estou com problemas, geralmente encontro uma saída". Quanto maior a pontuação, maior a autoeficácia.

Por fim, a Escala de Lócus de Controle da Saúde (MHLC), traduzida para versão brasileira (Rodríguez-Rosero, Ferriani, \& Dela Coleta, 2002), foi utilizada para análise da percepção de controle dos participantes. Nela estão presentes 18 itens que mensuram a atribuição de casualidade de sua saúde a fatores externos ou internos. Essa escala mensura três dimensões diferentes de atribuição de causalidade, cada uma dividida em 6 itens, na qual avalia: a internalidade, por exemplo, "Eu posso controlar a minha saúde"; a externalidade "outros poderosos", como por exemplo "Quem controla minha saúde são os médicos" e a externalidade "ao acaso", "Se fosse pelo destino, eu teria saúde". Com relação à consistência interna, o alfa de Cronbach encontrado para essa versão situa-se entre 0,51 e 0,78, considerado aceitável. Cada item é respondido em respostas do tipo Likert de cinco pontos.

\section{Procedimentos}

Os participantes foram abordados individualmente, e a aplicação das escalas ocorreu em forma de entrevista em uma sala reservada, quando disponível, ou em um local mais afastado das outras pessoas, para que fossem preservadas as informações obtidas dos participantes. Foi entregue o Termo de Consentimento Livre e Esclarecido (TCLE), a fim de esclarecer o ca- 
ráter confidencial de suas respostas e obter permissão para o uso científico das informações coletadas. Cabe ressaltar que esta pesquisa foi aprovada pelo Comitê de Ética em Pesquisa com Seres Humanos da Universidade Federal de Sergipe (CAAE 37689314.5.0000.5546).

Para a realização da coleta de dados, foi contatada anteriormente a Unidade Básica de Saúde (UBS) de Campo do Brito, para fins de obtenção da permissão para a realização de parte do estudo com os pacientes dentro da unidade. Também foram abordados indivíduos em suas residências, na Academia da Cidade e nas ruas. Ao serem questionados sobre o quadro hipertensivo com uso de medicamentos e confirmarem, os indivíduos foram convidados a participar da pesquisa. O tempo médio de aplicação de cada questionário foi de 15 minutos.

\section{Análise de Dados}

As respostas foram analisadas com o auxílio do programa SPSS (v. 22). Inicialmente foi realizada a análise exploratório-descritiva, obtendo-se as frequências percentuais, desvios padrões, os limites e as médias. As variáveis contínuas foram divididas em dois grupos a partir da mediana e as variáveis nominais foram agrupadas de acordo com os estratos de menor quantidade, separando-os em apenas dois grupos.

Executou-se a análise de Regressão Logística hierárquica (método Forward Wald) com a variável dependente Adesão ao Tratamento ( 0 = abaixo de seis pontos; $u m=$ de seis a oito pontos). Os dados referentes ao questionário sociodemográfico e aos dados clínicos (primeiro bloco), bem como os construtos do estudo (otimismo, autoeficácia, lócus interno, lócus externo ao acaso e lócus externo "outros poderosos" no segundo bloco) foram adicionados como variáveis independentes.

\section{Resultados}

Participaram desta pesquisa 49 homens e 51 mulheres, com média de idade de 50,3 anos (Desvio-Padrão = 10,75) e mediana em 49 anos (Mínimo = 32 e Máximo = 82). Sessenta e quatro por cento da amostra teve escolaridade até o ensino fundamental completo, $69 \%$ vivia com um companheiro e $62 \%$ da amostra ficou abaixo ou igual à mediana de 8 pontos de religiosidade. Quanto à renda mensal, 50\% recebiam abaixo ou igual a $R \$ 1630,00$. Quanto à autopercepção de saúde, 54\% pontuou até 6 (mediana), considerando-se que 0 correspondia a se perceber como doente e 10 pontos a se sentir saudável no momento da coleta de dados. O tempo de diagnóstico da HAS teve mediana de 6 anos, com 53\% até essa pontuação. Apenas 5\% se declararam tabagistas, $71 \%$ afirmaram não fazer uso regular de bebida alcoólica e $62 \%$ realizavam atividade física regularmente.

Com relação à adesão ao tratamento, a escala de Morisky (2008) originalmente interpreta pontuações abaixo de 6 como indicadores de baixa adesão. Como na amostra deste estudo a pontuação da maioria expressiva (85\%) dos participantes ficou abaixo do ponto de corte para estabelecimento de alta adesão (apenas 15\% com pontuação igual a 8), optou-se por categorizar os participantes em 2 grupos a partir da média adesão, ou seja, 6 pontos. 0 grupo de mais alta adesão corresponde a junção dos grupos de média ( 6 a 7 pontos) e alta adesão (8 pontos) conforme a proposta de Morisky (2008). Assim, o grupo de baixa adesão seguiu o mesmo critério estabelecido por Morisky. 
Tendo sido feito tal esclarecimento, detectou-se que 54\% da amostra pontuou até $<6$ pontos na escala, sendo então classificados como o grupo com baixa adesão. Os demais $46 \%$ pontuaram mais do que $\geq 6$ pontos, sendo então o grupo com média e alta adesão para a presente pesquisa. Em relação ao otimismo, a mediana foi de 17,5 pontos, e o agrupamento em dois estratos dividiu a amostra em 50\%. Quanto à autoeficácia, a mediana foi de 32 pontos, e 53\% da amostra pontuou até esse valor. Na análise do lócus de controle, as medianas e maior proporção dos grupos para o lócus interno, acaso e outros poderosos foram: 23,5 pontos (50\%), 19 pontos (60\%, até esse valor) e 23 pontos (58\%, até esse valor), respectivamente.

$\mathrm{Na}$ análise de regressão logística, apareceram como variáveis explicativas com significância estatística a atividade física ( $\operatorname{Sim}, O R=8,0, p<0,001$ ), bebida alcoólica (Não, $O R=5,3$, $p=0,005$ ), lócus de controle "acaso" (grupo acima de 19 pontos, $O R=4,5, p<0,008$ ) e a autoeficácia (grupo acima de 32 pontos, $O R=2,7, p=0,044$ ). Não foram estatisticamente significativas as variáveis sexo $(p=0,794)$, idade $(p=0,389)$, escolaridade $(p=0,312)$, relacionamento conjugal $(p=0,969)$, religiosidade $(p=0,279)$, renda média familiar $(p=0,640)$, autopercepção de saúde $(p=0,121)$, tempo de diagnóstico $(p=0,501)$, tempo de medicação $(p=0,606)$ e atendimento em urgência ou emergência no ano anterior $(p=0,928)$. O tabagismo não foi incluído na regressão, pois foi afirmado por apenas $5 \%$ da amostra, e tal proporção inviabilizou a inserção nos blocos de análise. Além dessas, o lócus de controle interno $(p=0,166)$, o lócus externo outros poderosos $(p=0,145)$ e o otimismo $(p=0,519)$ também não exibiram significância estatística. Na Tabela 1, estão expostos os resultados das variáveis com significância estatística na explicação da adesão ao tratamento.

Tabela 1

Regressão Logística para a Adesão ao Tratamento de Pessoas Hipertensas, Campo do Brito, Sergipe, $2017(n=100)$

\begin{tabular}{|c|c|c|c|c|}
\hline Variáveis & Categoria (F\%) & $\begin{array}{c}\text { Sig. } \\
(p<0,05)\end{array}$ & $\begin{array}{c}\operatorname{Exp}(\mathrm{B}) \\
\text { OddsRatio }\end{array}$ & IC95\% \\
\hline \multirow{2}{*}{ Uso de bebida alcoólica } & Não faz uso (71\%) & 0,005 & 5,316 & $1,666-16,968$ \\
\hline & Faz uso (29\%) & - & 1 & - \\
\hline \multirow{2}{*}{ Atividade física } & Pratica (62\%) & $<0,001$ & 8,043 & $2,599-24,887$ \\
\hline & Não pratica (38\%) & - & 1 & - \\
\hline \multirow{2}{*}{ Autoeficácia } & Acima da mediana (47\%) & 0,044 & 2,715 & $1,026-7,184$ \\
\hline & Até a mediana (32 pontos) (53\%) & - & 1 & - \\
\hline \multirow{2}{*}{ Lócus externo 'acaso' } & Acima da mediana (42\%) & 0,008 & 4,528 & $1,488-13,783$ \\
\hline & Até a mediana (19 pontos) (58\%) & - & 1 & - \\
\hline \multicolumn{5}{|c|}{ Notas. Variável dependente (1): média e alta adesão ao tratamento (6 a 8 pontos) $=46 \%(n=46)$. } \\
\hline \multicolumn{5}{|c|}{$\begin{array}{l}\text { F\% = frequência percentual; } n=\text { número de sujeitos; OR= razão de chances (OddsRatio); IC95\%= intervalo de } \\
\text { confiança de 95\%; } p=\text { significância estatística.-2/l Inicial = 113,607,-2/I Final = 102,436; Omnibus test= 33,552, } \\
p<0,001 ; \text { Nagelkerke } R=0,400 \text { (40\% de variância explicada); Hosmer \& Lemeshow } X^{2}=5,524, p=0,596 \text {; Per- } \\
\text { centual de casos corretamente preditos: } 80 \% \text {. }\end{array}$} \\
\hline
\end{tabular}




\section{Discussão}

O objetivo principal desta pesquisa foi analisar a relação entre o otimismo, a autoeficácia e o lócus de controle na adesão ao tratamento de hipertensos, bem como identificar os principais preditores da adesão, considerando as variáveis sociodemográficas, clínicas e relativas a esses construtos psicológicos. Diante disso, viu-se que as variáveis preditoras do grupo com média e alta adesão ao tratamento nessa amostra foram: atividade física, consumo de bebida alcoólica, autoeficácia e o lócus de controle externo "acaso". Especificamente quanto ao otimismo, não houve significância na explicação da adesão.

Em consonância com os achados da literatura (García-Pérez, Álvarez, Dilla, Gil-Guillén, \& Orozco-Beltrán, 2013; Girotto, Andrade, Cabrera, \& Matsuo, 2013), os participantes desta pesquisa que realizavam alguma atividade física chegaram a ter oito vezes mais chances de estarem entre aqueles com média e alta adesão, quando comparados àqueles que não praticam nenhum exercício. Sobre achados nessa mesma direção, Guedes e Lopes (2010) evidenciaram os benefícios físicos, psicológicos e comportamentais que o exercício físico possui na vida de hipertensos, dentre eles: redução da mortalidade e morbidade cardiovascular, diminuição da pressão arterial, melhora do padrão do sono e sentimento de independência em planejar sua própria caminhada. Outro achado nesse sentido é o do Aziz (2014), que encontrou evidências de que hipertensos que praticam atividades físicas de baixa intensidade (30 minutos de duração) e de forma regular (de 5 a 7 vezes por semana) conseguem reduzir a mortalidade cardiovascular em torno de $20 \%$. O presente estudo, portanto, reforça a importância da prática da atividade física como um aliado importante na adesão ao tratamento, já que os benefícios relacionados a ela vão além da manutenção do controle da pressão arterial e também estão relacionados a uma melhoria no condicionamento físico e psicológico dos hipertensos (Girotto et al., 2013).

O consumo de álcool também foi um fator preditivo de maior adesão entre os hipertensos, de modo que aqueles que relataram não ingerir bebidas alcoólicas tiveram aproximadamente cinco vezes mais chances de estarem no grupo com média e alta adesão. Chama também atenção o fato de que, no estudo de Girotto et al. (2013), a adesão ao tratamento em hipertensos foi associada ao não consumo regular de bebidas alcoólicas. Além disso, o Sistema de Vigilância de Fatores de Risco e Proteção para Doenças Crônicas por Inquérito Telefônico (Vigitel) apontou que o consumo excessivo de álcool costuma estar relacionado a outros comportamentos de risco à saúde, como tabagismo, sedentarismo e maior consumo de gordura (Brasil, 2013). O consumo do álcool eleva a pressão arterial de forma lenta e progressiva, e sua redução, além de diminuir a pressão, minimiza as chances de abandono do tratamento (Pessuto \& Carvalho, 1998). Logo, comportamentos de proteção à saúde, como prática de atividades física e menor consumo de álcool, demonstraram estar fortemente relacionados ao maior cuidado com o controle da pressão arterial no presente trabalho e, consoante a literatura (Girotto et al., 2013; Jesus et al., 2008; Medina, Lobo, Souza, Kanegusuko, \& Forjaz, 2010), confirma a relevância para que esses comportamentos sejam incentivados no tratamento da HAS.

A terceira variável preditora foi a autoeficácia, em que as pessoas com maiores índices tiveram quase três vezes mais chances de compor o grupo com média e alta adesão. A partir do instrumento aqui utilizado, não existe uma pontuação de corte na qual as pessoas são 
consideradas como pouco ou muito autoeficazes (Schwarzer, 2009). Para esse cálculo, o autor sugere utilizar uma divisão mediana da amostra e, a partir daí, estabelecer um ponto de corte. Neste trabalho, tal ponto de corte foi de 32 pontos. De acordo com Schwarzer (2009), a média de autoeficácia em várias amostras está em torno de 29, o que é um pouco abaixo da pontuação deste estudo. Na investigação de Castro et al. (2010), com adultos de 18 a 40 anos com doenças crônicas, a média de autoeficácia correspondeu a 33,2, semelhante ao desta pesquisa. De modo similar, Avelino (2014) encontrou em pacientes com artrite reumatoide um valor médio de 31,8 pontos. Distintamente, Mata, Carvalho, Gomes, Silva, e Pereira (2015) encontraram em pacientes que se submeteram à cirurgia de prostatectomia radical, valor médio de autoeficácia de 39 pontos.

Segundo Bandura (2004), uma alta percepção de autoeficácia influencia a saúde de duas formas. Na primeira, ela mantém a crença de que o indivíduo é capaz de lidar com eventos estressores relativos ao adoecimento, enquanto que, na segunda, ela faz com que a pessoa considere os efeitos de mudança de hábitos, emita comportamentos de promoção à saúde e consiga manter os hábitos que conseguiu atingir. Dessa forma, ao ter a crença e habilidade em sua capacidade de lidar com os estressores, as pessoas são motivadas a executar ações de proteção à saúde, evitar os comportamentos de risco e de obter melhores resultados terapêuticos, facilitando a adesão ao tratamento (Bandura, 2004; Lippke, Wiedemann, Ziegelmann, Reuter, \& Schwarzer, 2009; Rabelo \& Cardoso, 2007). Assim, junto aos hipertensos participantes desta pesquisa, viu-se que a autoeficácia se mostrou como uma variável que pode influenciar de forma positiva na adesão ao tratamento.

Por fim, ao analisar o resultado de lócus de controle e seu impacto sobre a adesão, foi encontrado que os participantes com maiores pontuações no lócus de controle "acaso" exibiram cerca de quatro vezes e meia mais chances de compor o grupo com média e alta adesão. De maneira geral, a literatura associa indivíduos com percepções externas de sua saúde a comportamentos mais negativos e menos aderentes (Helmer, Krämer, \& Mikolajczyk, 2012; Straub, 2014), além de serem mais propícios a adotarem práticas pouco saudáveis, pois são menos ativos no processo de tratamento hipertensivo.

Devido à discrepância entre o que revela a ideia inicial da influência da atribuição de causalidade e os dados desta investigação, é necessário destacar que, apesar dos achados em geral associarem pessoas com percepção interna de controle com características mais positivas em saúde, há casos em que uma visão mais externalizante de controle de sua doença pode ser uma estratégia de defesa mais adaptável que a primeira, mecanismo de amparo conhecido como externo defensivo (Rotter, 1966). Dessa forma, há contextos particulares em que o externo defensivo seja a melhor estratégia a ser adotada, como em situações em que o indivíduo deseja minimizar a culpa ou fracasso de determinado comportamento ou situação vivenciada. Assim, atribuir o controle da saúde ao acaso, embora possa parecer adverso a princípio, pode indicar uma redução na autoculpabilização a respeito de eventuais desfechos negativos que ocorram em termos de saúde e doença dos próprios indivíduos.

Outra possibilidade é a relação entre a ideia de acaso e a superstição de ter sorte como um fator decisivo quanto a ter boa saúde, ou não, na condição de hipertenso. Nessa linha, há experimentos que apontam para a existência de correlação negativa das crenças de ter boa sorte e a sintomatologia depressiva em adultos, o que viria a ocorrer por meio da alimen- 
tação de uma ilusão positiva de controle, autoconfiança e otimismo frente às adversidades (Day \& Maltby, 2003). Além desses indícios, há também experimentos psicológicos que encontraram que pessoas que foram incentivadas a ativar a superstição de "terem maior sorte" diante de tarefas motoras e cognitivas tenderam a mobilizar maiores níveis de autoeficácia, com uma significativa melhoria de desempenho nessas tarefas em comparação a um grupo controle em que tais superstições não foram ativadas (Damisch, Stoberock, \& Mussweiler, 2010).

Como os achados da presente pesquisa se basearam em instrumentos de autorrelato para a mensuração do lócus de controle e da adesão, parece razoável cogitar que essa ilusão positiva e certa desculpabilização devido ao fato de ter ou não sorte possam ter influenciado, em alguma medida, a relação positiva entre acaso e adesão ao tratamento deste estudo. Considerando-se ainda que a relação entre tais crenças de acaso e desfechos em saúde também foi aventada nesses dois estudos (Damisch et al., 2010; Day \& Maltby, 2003), talvez se possa aplicar tal hipótese ao entendimento do porquê hipertensos com maiores pontuações no lócus de controle acaso, mediante uma expectativa de ter alguma sorte na condução de seu tratamento, exibiram uma mais alta adesão na corrente investigação.

De modo complementar, no que tange à importância de se considerar o lócus externo como algo passível de assumir um caráter positivo na saúde, há pesquisas que propõem que indivíduos que convivem com doenças crônicas - tais como a HAS e HIV - tendem a depositar maior confiança na equipe de saúde e nos familiares. Com isso, tornam-se mais dispostos a deixarem-se submeter ao tratamento ao se sentirem mais acolhidos e perceberem menos obstáculos internos frente ao tratamento terapêutico e clínico (Almeida, Pereira, \& Fontoura, 2012; Nazareth et al., 2015).

Em consideração ao que foi detectado neste trabalho, no caso de doenças crônicas como a HAS, acredita-se que uma postura mais externalizante em saúde pode também ampliar a adesão ao tratamento hipertensivo, constituindo-se, então, como uma particularidade contextual do funcionamento do lócus de controle em saúde. Aqui se constatou que atribuições voltadas ao lócus externo acaso parecem aumentar a motivação do paciente em continuar o tratamento de controle da hipertensão, ajudando a diminuir, por exemplo, a autopercepção negativa do indivíduo.

Em suma, os resultados desta pesquisa sugerem que a presença de certa desculpabilização (alta pontuação em lócus de controle acaso), sem haver necessariamente desresponsabilização para com o tratamento (mais alta pontuação na escala de adesão), tem importante capacidade de explicação da adesão ao tratamento nesta amostra. Tal como afirmado por Day e Maltby (2003), vê-se que atribuições e crenças de acaso ou sorte nem sempre refletem baixos níveis de adaptação, ou seja, não são intrinsecamente negativas.

Como limitações da pesquisa, a principal delas é pouco quantidade de participantes classificadas no grupo de alta adesão (8 pontos), o que exigiu agrupar média e alta adesão em um grupo. De qualquer modo, Morisky et al. (2008) considerava a adesão como satisfatória a partir da média adesão, ou seja, os achados desta pesquisa não destoam da proposta inicial do autor da escala. Embora tenhamos considerado que 15\% foi uma proporção baixa no grupo com alta adesão, vale destacar que, nos estudos de Morisky et al. (2008) e no de OliveiraFilho et al. (2012), também foram encontradas proporções abaixo de 20\%. Considerando 
que a regressão logística demandaria um número maior de pessoas para a comparação entre grupos, optamos por unir os grupos de média e alta adesão, grupos estes que são considerados por Morisky como aderentes. Ademais, salienta-se novamente que as informações então obtidas foram autorreferidas, o que pode ter gerado erros decorrentes de memória ou de outras distorções e, por isso, alguns resultados podem estar sub ou superestimados. Sugestões para próximas investigações, além do aprofundamento da influência do lócus de controle acaso sobre a saúde, envolvem associação de outras variáveis psicológicas à temática, tais como a percepção supersticiosa de ter sorte frente às adversidades. Ademais, julga-se pertinente incentivar que futuros estudos avaliem como essas variáveis afetam o processo de adesão ao tratamento não só dos hipertensos, mas também de outras enfermidades de alta prevalência e comumente comórbidas à hipertensão, a exemplo do diabetes.

Finalmente, evidencia-se que ainda são escassos os trabalhos sobre aspectos psicológicos da adesão ao tratamento de hipertensos, principalmente quando se trata de Estados e Municípios de menor porte no Brasil; algo diferencial nesta pesquisa. Através deste estudo, espera-se motivar novas pesquisas com essa temática, assim como a elaboração de estratégias que aumentem a adesão ao tratamento, levando-se em conta a influência de aspectos psicológicos sobre os comportamentos em saúde.

\section{Referências}

Afonso, L. M. (2004). Acerca del concepto de adherencia terapéutica. Revista Cubana de Salud Pública, 30(4).

Almeida, P., Pereira, M. G., \& Fontoura, M. (2012). Variáveis individuais e familiares na adesão ao tratamento, controle metabólico e qualidade de vida em adolescentes com diabetes tipo 1. Sociedade Brasileira de Psicologia Hospitalar, 15, 60-82.

Araújo, G. B. S., \& Garcia, T. R. (2006). Adesão ao tratamento anti-hipertensivo: Uma análise conceitual. Revista Eletrônica de Enfermagem, 8(2), 259-272.

Avelino, R. P. (2014). Autoeficácia de pacientes com artrite reumatoide inicial e crônica: Uma análise comparativa (Monografia do curso de Terapia Ocupacional, Universidade de Brasília, Brasília, DF).

Aziz, J. L. (2014). Sedentarismo e hipertensão arterial. Revista Brasileira de Hipertensão, 21(2), 75-82.

Bandeira, M., Bekou, V., Lott, K. S., Teixeira, M. A., \& Rocha, S. S. (2002). Validação transcultural do Teste de Orientação da Vida (TOV-R). Estudos de Psicologia, 7(2), 251-258.

Bandura, A. (1977). Self-Efficacy: Toward a unifying theory of behavioral change. Psychological Review, 84(2), 191-215.

Bandura, A. (2004). The growing primacy of perceived efficacy in human self-development, adaptation and change. In M. Salanova, R. Grau, I. M. Martinez, E. Cifre, S. Llorens, \& M. García-Renedo (Eds.), Nuevos horizontes en la investigación sobre autoeficacia (pp. 3351). Castellón: Colección Psyique.

Baptista, M. N., Santos, K. M., \& Dias, R. R. (2006). Auto-eficácia, lócus de controle e depressão em mulheres com câncer de mama. Psicologia Argumento, 24(44), 27-36.

Bastianello, M. R., \& Hutz, C. S. (2015). Do otimismo explicativo ao disposicional: A perspectiva da Psicologia Positiva. Psico-USF, 20(2), 237-247. doi: 10.1590/1413-82712015200205 
Bernard, J. E. R. (2011). Variables psicosociales implicadas en el mantenimiento y control de la diabetes mellitus: Aspectos conceptuales, investigaciones y hallazgos. Revista Electrónica de Psicología Iztacala, 14(2), 126-162.

Brasil. Ministério da Saúde (MS). (2013). Vigitel Brasil 2012: Vigilância de fatores de risco e proteção para doenças crônicas por inquérito telefônico. Secretaria de Vigilância em Saúde, Departamento de Vigilância de Doenças e Agravos não Transmissíveis e Promoção de Saúde. Brasília.

Brito, T. M., \& Giorgi, D. M. A. (2012). Como diagnosticar e tratar hipertensão arterial sistêmica. Revista Brasileira de Medicina, 69(12), 6-15.

Carver, C. S., \& Connor-Smith, J. (2010). Personality and coping. Annual Review of Psychology, 31, 679-704. doi: 10.1146/annurev.psych.093008.100352

Castro, E. K., Ponciano, C. F., \& Pinto, D. W. (2010). Autoeficácia e qualidade de vida de jovens adultos com doenças crônicas. Revista Aletheia, 31, 137-148.

Damisch, L., Stoberock, B., \& Mussweiler, T. (2010). Keep your fingers crossed! How superstition improves performance. Psychological Science, 21(7), 1014-1020. doi: 10.1177/0956797610372631

Day, L., \& Maltby, J. (2003). Belief in good luck and psychological well-being: The mediating role of optimism and irrational beliefs. The Journal of Psychology: Interdisciplinary and Applied, 137(1), 99-110. doi: 10.1080/00223980309600602

García-Pérez, L. E., Álvarez, M., Dilla, T., Gil-Guillén, V., \& Orozco-Beltrán, D. (2013). Adherence to therapies in patients with type 2 diabetes. Diabetes Ther, 4, 175-194. doi: 10.1007/ s13300-013-0034-y

Gaspar, T., Ribeiro, J. L. P., Matos, M. G., Leal, I., \& Ferreira, A. (2009). Optimismo em crianças e adolescentes: Adaptação e validação do LOT-R. Psicologia Reflexão e Crítica, 22(3), 439446. doi: 10.1590/S0102-79722009000300015

Girotto, E., Andrade, S. M., Cabrera, M. A. S., \& Matsuo, T. (2013). Adesão ao tratamento farmacológico e não farmacológico e fatores associados na atenção primária da hipertensão arterial. Ciência \& Saúde Coletiva, 18(6), 1763-1772. doi: 10.1590/ S1413-81232013000600027

Guedes, N. G., \& Lopes, M. V. O. (2010). Exercício físico em portadores de hipertensão arterial: Uma análise conceitual. Revista Gaúcha de Enfermagem, 31(2), 367-374. doi: 10.1590/S1983-14472010000200023

Hair, J. F., Black, W. C., Babin, B. J., Anderson, R. E., \& Tatham, R. L. (2009). Análise multivariada de dados (6a ed.). Porto Alegre: Bookman.

Helmer, S. M., Krämer, A., \& Mikolajczyk, R. T. (2012). Health-related locus of control and health behaviour among university students in North Rhine Westphalia, Germany. BMC Research Notes, 5(1), 1-8. Disponível em https://bmcresnotes.biomedcentral.com/track/ pdf/10.1186/1756-0500-5-703

Jesus, E. S., Augusto, M. A. O., Gusmão, J., Mion Júnior, D., Ortega, K., \& Pierin, A. M. G. (2008). Perfil de um grupo de hipertensos: Aspectos biossociais, conhecimentos e adesão ao tratamento. Acta Paulista de Enfermagem, 21, 59-65.

Joint National Committee on Prevention, Detection, Evaluation, and Treatment of High Blood Pressure. (2004). The seventh report of the Joint National Committee on Prevention, Detection, Evaluation, and Treatment of High Blood Pressure. JAMA, 289, 2560-2571. 
Lima, T. M., Meiners, M. M. M. A., \& Soler, O. (2010). Perfil de adesão ao tratamento de pacientes hipertensos atendidos na Unidade Municipal de Saúde de Fátima, em Belém, Pará, Amazônia, Brasil. Pan-Amazônica de Saúde, 1(2), 113-120. doi: 10.5123/ S2176-62232010000200014

Lippke, S., Wiedemann, A. U., Ziegelmann, J. P., Reuter, T., \& Schwarzer, R. (2009). Selfefficacy moderates the mediation of intentions into behavior via plans. American Journal of Health Behaviour, 33(5), 521-529. Disponível em http://userpage.fu-berlin.de/ health/ hapa/lippke2009.pdf

Mata, L. R. F., Carvalho, E. C., Gomes, C. R. G., Silva, A. C., \& Pereira, M. G. (2015). Autoeficácia e morbidade psicológica no pós-operatório de prostectomia radical. Revista LatinoAmericana de Enfermagem, 23(5), 806-813. doi: 10.1590/0104-1169.0456.2618

Medina, F. L., Lobo, F. S., Souza, D. R., Kanegusuku, H., \& Forjaz, C. L. M. (2010). Atividade física: Impacto sobre a pressão arterial. Revista Brasileira de Hipertensão, 17(2), 103-106.

Morisky, D. E., Ang, A., Krousel-Wood, M., \& Ward, H. J. (2008). Predictive validity of a medication adherence measure in an outpatient setting. The Journal of Clinical Hypertension, 10(5), 348-354.

Morowatisharifabad, M. A., Ghofranipour, F., Heidarnia, A., \& Ruchi, G. B. (2006). Self-efficacy and health promotion behaviors of older adults in Iran. Social Behavior and Personality, 34(7), 759-768.

Nakamoto, A. Y. K. (2012). Hipertensão arterial sistêmica. Revista Brasileira de Medicina, 69(4), 78-86.

Nazareth, M., Richards, J., Javalkar, K., Haberman, C., Zhong, Y., Rak, E., Jain, N., Ferris, M., \& Tilburg, M. A. L. (2015). Relating health locus of control to health care use, adherence, and transition readiness among youths with chronic conditions. Preventing Chronic Disease, 13(7). doi: 10.5888/pcd13.160046

Oliveira-Filho, A. D., Barreto-Filho, J. A., Neves, S. J. F., \& Lyra, D. P., Jr. (2012). Relação entre a escala de adesão terapêutica de oito itens de Morisky (MMAS-8) e o controle da pressão arterial. Arquivos Brasileiros de Cardiologia, 99(1), 649-658. doi: 10.1590/ S0066-782X2012005000053.

Omeje, O., \& Nebo, C. (2011). The influence of control on adherence to treatment regimen among hypertensive patients. Patient Preference and Adherence, 5, 141-148. doi: 10.2147/ PPA.S15098

Organização Mundial da Saúde (OMS). (2013). A global brief on hypertension. Geneve: World Health Organization.

Organização Mundial da Saúde (OMS). (2014). Health statistics and information systems. Geneve: World Health Organization.

Ortega, K. C., Silva, G. V., \& Mion, D., Jr. (2006). Hipertensão arterial sistêmica. Revista Brasileira de Medicina, 63, 19-28.

Ortiz, M. P., \& Ortiz, E. P. (2007). Psicología de la salud: Una clave para comprender el fenómeno de la adherencia terapéutica. Revista Médica de Chile, 135(5), 647-652. doi: 10.4067/S0034-98872007000500014

Péres, D. S., Magna, J. M., \& Viana, L. A. (2003). Portador de hipertensão arterial: Atitudes, crenças, percepções, pensamentos e práticas. Revista de Saúde Pública, 37(5), 635-642. doi: 10.1590/S0034-89102003000500014 
Pessuto, J., \& Carvalho, E. C. (1998). Fatores de risco em indivíduos com hipertensão arterial. Revista Latino-Americana de Enfermagem, 6, 33-39.

Rabelo, D. F., \& Cardoso, C. M. (2007). Auto-eficácia, doenças crônicas e incapacidade funcional na velhice. Psico-USF, 12, 75-81.

Rodrigues, D. N., \& Pereira, C. A. A. (2007). A percepção de controle como fonte de bemestar. Estudos e Pesquisas em Psicologia, 7(3), 541-556.

Rodríguez-Rosero, J. E., Ferriani, M. G. C., \& Dela Coleta, M. F. (2002). Escala de lócus de controle da saúde - MHLC: Estudos de validação. Revista Latino-Americana de Enfermagem, 10(2), 179-184. doi: 10.1590/S0104-11692002000200009

Rotter, J. B. (1966). Generalized expectancies for internal versus external control of reinforcement. Psychological Monographs, 80(1), 1-28. doi: 10.1037/h0092976

Schwarzer, R. (2009). Everything you wanted to know about the General Self-efficacy Scale but were afraid to ask. Documentation of the General Self-Efficacy Scale. Disponível em http://userpage.fu-berlin.de/health/faq_gse.pdf

Shepperd, J. A., Maroto, J. J., \& Pbert, L. A. (1996). Dispositional optimism as a predictor of health changes among cardiac patients. Journal of Research in Personality, 30, 517-534.

Sociedade Brasileira de Cardiologia. (2016). VII Diretrizes Brasileiras de Hipertensão Arterial. Arquivos Brasileiros de Cardiologia, 107(3), 21-103.

Souza, I., \& Souza, M. A. (2004). Validação da escada de auto-eficácia geral percebida. Revista da Universidade Rural do Rio de Janeiro Série Ciências Humanas, 26(1-2), 12-17.

Straub, R. O. (2014). Psicologia da saúde: Uma abordagem biopsicossocial (3a ed.). Porto Alegre: Artmed.

Wood, R., \& Bandura, A. (1989). Impact of conceptions of ability on self-regulatory mechanisms and complex decision making. Journal of Personality and Social Psychology, 56(3), 407-415.

Recebido: 18/02/2018

Última revisão: 18/06/2018

Aceite final: 19/06/2018

\section{Sobre os autores:}

Lucila Moraes Chaves Santos - Graduada em Psicologia pela Universidade Federal de Sergipe (UFS). E-mail: lucilamoraescs@gmail.com, Orcid: http://orcid.org/0000-0003-1819-2293

Laís Gabriela Rocha Almeida - Mestranda em Psicologia Clínica e da Saúde na Universidade da Beira Interior (Portugal). Graduada em Psicologia pela Universidade Federal de Sergipe (UFS). E-mail: laisgabriela.psi@hotmail.com, Orcid: http://orcid.org/0000-0002-3747-0183

André Faro - Pesquisador visitante e Pós-Doutor pela Johns Hopkins University (Bloomberg School of Public Health). Doutor em Psicologia pela Universidade Federal da Bahia (UFBA). Professor Adjunto do Departamento de Psicologia e Programa de Pós-Graduação em Psicologia da Universidade Federal de Sergipe (UFS). E-mail: andre.faro.ufs@gmail.com, Orcid: http://orcid.org/0000-0002-7348-6297 